The Astrophysical Journal, 339:904-918, 1989 April 15

C) 1989. The American Astronomical Society. All rights reserved. Printed in U.S.A.

\title{
AN ANALYSIS OF THE DISTRIBUTION OF GLOBULAR CLUSTERS WITH POSTCOLLAPSE CORES IN THE GALAXY
}

\author{
DAVID F. CHERNOFF ${ }^{1}$ \\ Center for Radiophysics and Space Research, Cornell University \\ AND \\ S. DJORGOVSKI ${ }^{2}$ \\ California Institute of Technology \\ Received 1988 May 6; accepted 1988 September 20
}

\begin{abstract}
We present a new compilation of structural parameters for Galactic globular clusters, based on the data from the complete survey published by Djorgovski, King, and collaborators in 1984, 1986, and 1987 and the literature. We find that the distribution of the post-core-collapsed (PCC) clusters is much more concentrated about the Galactic center than the distribution of the King model (KM) clusters. Within the KM family, a similar trend exists: centrally condensed KM clusters are found, on average, at smaller galactocentric radii. To deal with the problem of Galactic obscuration, we used a distance-independent analysis, similar to one developed and published by Frenk and White in 1982. We analyzed the shapes of the KM and PCC cluster systems; they are each consistent with a symmetrical distribution of the clusters about the Galactic center. At fixed distance from the center, the clusters at smaller heights above the plane (and thus the less inclined orbits) are marginally more concentrated. The data indicate that the more concentrated KM clusters tend to have higher luminosities. On the other hand, the PCC clusters are less luminous than the highest concentration KM clusters. The PCC clusters show signs of tidal distortions in their envelopes: their major axes tend to point toward the Galactic center; the KM clusters show no such effect. There is a very slight difference in the average metallicities of the two families, in the sense that PCC clusters have slightly lower metal abundances, but there is no significant trend with the concentration within the KM family.

The fact that some internal properties of clusters correlate well with global variables, such as the galactocentric radius, suggests that some external effects are important in cluster evolution. These include the average cluster density, set by the mean tidal field of the Galaxy, and, perhaps, the strength of tidal shocks.
\end{abstract}

Subject headings: clusters: globular — galaxies: The Galaxy — galaxies: structure — stars: stellar dynamics

\section{INTRODUCTION}

There have been a number of important advances in our understanding of globular cluster evolution in the past few years (cf. the volumes edited by Goodman and Hut 1985, Grindlay and Philip 1987, and the reviews by Elson, Hut, and Inagaki 1987 and Spitzer 1987 and references therein). On the observational side, increasingly accurate studies have established that cluster profiles in and near the cores are not always well fitted by King (1966) models, and instead may have substantial power-law regions. Although discrepancies between observations and King model fits had been noted before in the case of M15 (King 1975; Newell and O'Neil 1978), nearly complete and uniform studies of the Galactic cluster have been carried out recently which showed that approximately $20 \%$ of clusters differ significantly from King models (Djorgovski and King 1986; Djorgovski et al. 1986).

On the theoretical side, the problem of core collapse and subsequent evolution has been addressed by a number of authors. Hénon (1961) predicted that the core collapse should ultimately occur in all sufficiently concentrated clusters, and even suggested that the formation of central binaries may arrest the collapse. While significant uncertainties remain, it is now clear that core collapse does not represent a catastrophic

\footnotetext{
1 Presidential Young Investigator.

${ }^{2}$ Visiting Astronomer, Cerro Tololo Interamerican Observatory, NOAO, operated by AURA, Inc., under contract with the NSF.
}

event in the life of a cluster, but rather a normal point which many clusters will reach and pass through in the future, if they have not done so already. Specific models of collapse of isolated clusters, composed of single-mass stars, show that a power-law density profile develops over a large radial range during the collapse phase. How much of this power-law profile clusters retain during the period of core expansion is a key question. An interesting complication in the postcollapse phase is the occurrence of gravothermal oscillations (Bettwieser and Sugimoto 1984). Goodman's (1987) analysis of simple models with equal mass stars and a generic heating source shows that core oscillations involve only the inner $1 \%$ or $2 \%$ of the mass. The detailed behavior of the density profile is complicated, varying between $\rho \sim r^{-2.2}$ and $\rho \sim r^{-2}$ in the inner oscillating region. However, both the limitations in observational accuracy and especially the theoretical simplifications make these fine distinctions irrelevant for the present discussion. Therefore, it may be sufficient to identify core-collapsed clusters by the presence of substantial inner radial ranges of power-law behavior in the surface brightness.

Although this is a simple and attractive picture, there are a number of uncertainties. First, there are a host of possible complications associated with the reexpansion phase. The proper, detailed treatment of the cluster reexpansion is inevitably tied to the breakdown of the Fokker-Planck equation which the core collapse signals. It is uncertain whether current treatments which use the Fokker-Planck equation with effec- 
tive heating terms are adequate. It is also trivially true that a spectrum of stellar mass types is present, which doubtless modifies the rate of binary formation. Likewise, there is the issue of whether such a spectrum alters, in a significant fashion, the system's response to heating; in particular, it is unclear whether a continuum distribution of masses undergoes gravothermal oscillations as a single-component system does. Finally, there is the practical complication that the cluster light is dominated by the $\mathrm{K}$ giants, which do not necessarily trace the cluster mass. Realistic study of the internal distribution of stellar types has only just begun.

The plan of the paper is as follows. We begin by discussing the observed properties of King model (KM) and PCC clusters in $\S$ II. We assume that the classification of cluster type (PCC vs. KM) is nearly independent of selection effects. A PCC cluster is fundamentally different from a King model; Djorgovski and King (1986) have argued that the observational data are sufficiently accurate to differentiate the alternatives unambiguously for practically all known clusters. A nearly complete catalog of clusters with classifications has been assembled. In $\S$ III we compare the Galaxy-wide distribution of PCC clusters to normal King model clusters. We have made use of the technique of Frenk and White (1982), who studied properties of the cluster distribution which could be inferred from the two-dimensional distribution on the sky, without introducing uncertainties associated with the cluster distance measurements. We show that the projected cluster sample, as a whole, is distributed approximately symmetrically about the Galactic center on the sky, i.e., without a gross flattening. The situation for the PCC clusters is less certain: these clusters may be members of a nonspherical distribution or they may be subject to significant observational biases. The radial distribution of clusters about the Galactic center on the sky shows that there is a very strong tendency for PCC clusters to be located near the Galactic center. Finally, although the observer selection effects are less well understood in the case of the threedimensional distribution, we show that there is an increase in the fraction of PCC clusters near the Galactic center. We have also included an analysis of the trends in mass, concentration, and metalicity with galactocentric position. These trends are suggestive, but none are nearly as strong as the trend towards a centrally increasing fraction of PCC clusters. In § IV we make the assumption that PCC clusters are cluster which have their cores collapsed and explore evolutionary scenarios which could explain the trend using a simplified treatment of globular cluster evolution. This approximate treatment, which only applies to clusters on circular orbits, includes a number of the most significant mechanisms of precollapse cluster evolution: mass loss across a tidal boundary, stellar mass loss by evolving stars, relaxation via evaporation, and tidal shocking by disk passages. A number of shortcomings of the approach are emphasized.

\section{KING MODELS AND PCC CLUSTERS}

Surface brightness profiles of globular clusters have been used to classify clusters as post-core-collapsed (PCC) or King model (KM) states as discussed by Djorgovski and King (1986). PCC clusters are distinguished from KM clusters by having an extensive power-law distribution of light in their central parts. The data come from the Berkeley globular clusters survey (Djorgovski and King 1984, 1986; Djorgovski and Penner 1985; Djorgovski et al. 1986; King et al. 1989), supplemented by the profiles from Kron, Hewitt, and Wasserman (1984), the star counts by King et al. (1968) and Peterson (1976), and several other sources, summarized in the report by Djorgovski and King (1986). All available profiles for each cluster, and a set of King (1966) models were plotted on the same log-log scale, and the King model curves were fitted by eye. This was done deliberately, rather than through an automatic procedure, since it was often necessary to make complex decisions and judgments about the radial range to be trusted for each profile, about recognizing and ignoring bad data points, the estimates of quality and weighting of different profiles of the same cluster, etc. Generally, the new CCD data were given the highest weight, since they contain the most information about the central parts of clusters, which are of the primary interest here. The angular core radius $r_{c}$, the angular tidal radius $r_{t}$, and the King model concentration parameter $c=\log \left(r_{t} / r_{c}\right)$ were determined; the relevant results are listed in Table 1. From internal comparisons (independent estimates for the same cluster), and comparisons with the numbers published by other authors, we estimate that the average accuracy of our $c$ measurement is about $0.1-0.2$, and the measurements of $\log r_{c}$ about 0.1 . The clusters classified as having or probably having collapsed cores mainly follow the classification by Djorgovski and King (1986). We believe that our measurements for the sample supersede other similar compilations currently available in the literature, but we anticipate a better set of morphological parameters coming in the near future (King et al. 1989).

We begin by discussing in detail the distinction between PCC and KM clusters (see also Djorgovski and King 1986). All cluster observations have inner and outer angular radii which delimit the region of reasonable measurement accuracy of the cluster profile. The inner limit is set by the quality of seeing and, typically, $\theta_{\min } \sim 1$ "; the outer limit is set by the level of sky contamination and confusion of background and foreground stars complemented by the decreasing number density of cluster members. It is typically $\theta_{\max } \sim 150^{\prime \prime}$. Over the range of $\left[\theta_{\min }, \theta_{\max }\right]$, under optimum observing conditions, cluster profiles may be measured to an accuracy that is limited primarily by $N^{1 / 2}$ fluctuations in the number of red giants, which produce most of the light.

It is traditional to fit cluster profiles with King models. One scheme is to fit the profile over the range $\left[\theta_{\min }, \theta_{\max }\right]$ using a least-squares technique, weighting the points by the observationally determined uncertainties. The profile uncertainties (assumed to be $N^{1 / 2}$ in origin) are derived by dividing each annulus into several angular regions and then calculating the formal error about the whole annulus result (see Djorgovski 1987b). The fit for the projected King model has three parameters, a central surface density, $\sigma(0)$, an angular core size, $r_{c}$, and a concentration parameter $c=\log \left(r_{t} / r_{c}\right)$, where $r_{t}$ is the angular tidal radius. The fit is carried out by choosing $r_{c}$ and $c$, and then adjusting $\sigma_{\text {King }}(0)$ to minimize $\chi^{2}$ where

$$
\chi^{2}=\sum_{i}\left[\frac{\sigma\left(\theta_{i}\right)-\sigma_{\mathrm{King}}\left(\theta_{i}\right)}{\delta \sigma\left(\theta_{i}\right)}\right]^{2} .
$$

In the sum, $i$ runs over the data points with $\theta_{\min }<\theta_{i}<\theta_{\max }$, where $\sigma\left(\theta_{i}\right)$ is the measured value, $\delta \sigma\left(\theta_{i}\right)$ is the measured uncertainty and $\sigma_{\mathrm{King}}$ is the King model.

To illustrate the characteristic results, we have carried out the above procedure on NGC 6388, classified as a KM cluster, and Trz 2, classified as a PCC cluster. Figure 1 illustrates contours of the formal error in the fits of King models for NGC 6388 , with $\theta_{\min }=1 " .5$ and $\theta_{\max }=122^{\prime \prime}$, as a function of $c$ and $r_{c}$. 
TABLE 1

Observed Parameters for Individual Clusters

\begin{tabular}{|c|c|c|c|c|c|c|c|c|c|c|}
\hline Cluster & $c$ & $R / \mathrm{kpc}$ & $Z / \mathrm{kpc}$ & $\mathbf{M}_{V}$ & $\log M / M_{\odot}$ & $\log r_{c}$ & $\left\langle Q_{30}\right\rangle$ & $|\Delta P A|$ & Separ. & Morph. \\
\hline NGC 104 & 2.03 & 8.1 & 3.2 & -9.1 & 6.06 & 1.40 & 0.323 & $38^{\circ}$ & $65^{\circ}$ & $\mathrm{KM}$ \\
\hline NGC 288 & 1.00 & 12.1 & 8.2 & -5.1 & 4.45 & 1.85 & - & - & - & $\mathrm{KM}$ \\
\hline NGC 362 & 1.75 & 9.9 & 6.3 & -8.3 & 5.72 & 0.95 & 0.188 & $37^{\circ}$ & $69^{\circ}$ & PCC? \\
\hline NGC 1261 & 1.40 & 18.3 & 12.7 & -7.7 & 5.49 & 1.30 & - & $44^{\circ}$ & $90^{\circ}$ & $\mathrm{KM}$ \\
\hline Pal 1 & 1.50 & 20.3 & 4.5 & -2.5 & 3.43 & 0.95 & - & $68^{\circ}$ & $128^{\circ}$ & $\mathrm{KM}$ ? \\
\hline AM 1 & 0.70 & 118. & 86.5 & -4.5 & 4.23 & 1.30 & - & - & - & $\mathrm{KM}$ ? \\
\hline Eridanus & 1.10 & 90.2 & 55.9 & -4.1 & 4.06 & 1.20 & - & - & - & KM? \\
\hline Pal 2 & 1.70 & 22.2 & 2.1 & -7.2 & 5.28 & 0.70 & - & $53^{\circ}$ & $167^{\circ}$ & KM? \\
\hline NGC 1851 & 2.15 & 17.2 & 6.9 & -8.2 & 5.70 & 0.60 & - & $79^{\circ}$ & $111^{\circ}$ & $\mathbf{K M}$ \\
\hline NGC 1904 & 1.90 & 19.5 & 6.4 & -7.7 & 5.50 & 0.90 & - & $87^{\circ}$ & $126^{\circ}$ & PCC? \\
\hline NGC 2298 & 1.50 & 16.2 & 2.9 & -6.2 & 4.87 & 1.30 & - & $56^{\circ}$ & $113^{\circ}$ & KM \\
\hline NGC 2419 & 1.60 & 99.4 & 39.0 & -9.2 & 6.09 & 1.35 & - & $1^{\circ}$ & $155^{\circ}$ & $\mathbf{K M}$ \\
\hline NGC 2808 & 1.80 & 11.6 & 1.9 & -9.4 & 6.15 & 1.15 & 0.172 & $76^{\circ}$ & $78^{\circ}$ & $\mathrm{KM}$ \\
\hline Pal 3 & 1.40 & 91.5 & 58.7 & -4.6 & 4.25 & 1.45 & - & - & - & $\mathrm{KM}$ \\
\hline NGC 3201 & 1.35 & 9.5 & 0.8 & -7.1 & 5.26 & 1.80 & - & $57^{\circ}$ & $83^{\circ}$ & $\mathrm{KM}$ \\
\hline $\mathrm{Pal} 4$ & 0.80 & 96.2 & 88.6 & -5.7 & 4.69 & 1.70 & - & $-\overline{0}$ & - & KM \\
\hline NGC 4147 & 2.30 & 19.9 & 16.9 & -5.9 & 4.77 & 0.50 & - & $87^{\circ}$ & $94^{\circ}$ & KM? \\
\hline NGC 4372 & 1.10 & 7.6 & 0.8 & -6.2 & 4.89 & 2.00 & - & $66^{\circ}$ & $59^{\circ}$ & $\mathrm{KM}$ \\
\hline NGC 4590 & 1.60 & 10.1 & 5.6 & -6.7 & 5.10 & 1.60 & -0.043 & $7^{\circ}$ & $66^{\circ}$ & $\mathbf{K M}$ \\
\hline NGC 4833 & 1.10 & 7.4 & 0.8 & -7.6 & 5.47 & 1.85 & 0.017 & $87^{\circ}$ & $57^{\circ}$ & KM \\
\hline NGC 5024 & 1.60 & 19.2 & 18.2 & -8.6 & 5.86 & 1.35 & - & $22^{\circ}$ & $81^{\circ}$ & $\mathrm{KM}$ \\
\hline NGC 5053 & 0.80 & 16.7 & 15.5 & -6.1 & 4.84 & 2.10 & - & $24^{\circ}$ & $80^{\circ}$ & $\mathrm{KM}$ \\
\hline NGC 5139 & 1.70 & 7.0 & 1.3 & -9.4 & 6.16 & 2.25 & - & $2^{\circ}$ & $52^{\circ}$ & $\mathrm{KM}$ \\
\hline NGC 5272 & 1.85 & 12.6 & 10.2 & -8.8 & 5.94 & 1.50 & - & $22^{\circ}$ & $\mathbf{8 2}^{\circ}$ & $\mathrm{KM}$ \\
\hline NGC 5286 & 1.80 & 7.7 & 1.8 & -8.1 & 5.67 & 1.15 & 0.056 & $66^{\circ}$ & $49^{\circ}$ & $\mathrm{KM}$ \\
\hline NGC 5466 & 1.10 & 16.4 & 15.2 & -6.7 & 5.08 & 2.10 & - & $24^{\circ}$ & $78^{\circ}$ & $\mathrm{KM}$ \\
\hline NGC 5634 & 1.60 & 20.7 & 18.9 & -9.3 & 6.15 & 1.10 & - & $0^{\circ}$ & $52^{\circ}$ & $\mathrm{KM}$ \\
\hline NGC 5694 & 1.80 & 25.4 & 15.8 & -8.5 & 5.83 & 0.60 & 0.002 & $12^{\circ}$ & $41^{\circ}$ & $\mathrm{KM}$ \\
\hline NGC 5824 & 2.30 & 18.0 & 9.2 & -8.4 & 5.76 & 0.50 & 0.014 & $72^{\circ}$ & $35^{\circ}$ & $\mathbf{K M}$ \\
\hline Pal 5 & 1.20 & 16.5 & 15.4 & -5.0 & 4.41 & 2.20 & - & - & - & KM? \\
\hline NGC 5897 & 1.20 & 6.7 & 6.0 & -6.9 & 5.18 & 1.90 & - & $4^{\circ}$ & $34^{\circ}$ & $\mathrm{KM}$ \\
\hline NGC 5904 & 1.40 & 6.6 & 5.5 & -8.7 & 5.91 & 1.40 & 0.085 & $11^{\circ}$ & $47^{\circ}$ & KM \\
\hline NGC 5927 & 1.60 & 5.1 & 0.7 & -8.0 & 5.61 & 1.40 & 0.426 & $0^{\circ}$ & $34^{\circ}$ & $\mathrm{KM}$ \\
\hline NGC 5946 & - & 5.1 & 0.7 & -7.3 & 5.32 & - & 0.140 & $20^{\circ}$ & $33^{\circ}$ & PCC \\
\hline NGC 5986 & 1.30 & 4.7 & 2.4 & -8.2 & 5.67 & 1.50 & - & $70^{\circ}$ & $26^{\circ}$ & $\mathrm{KM}$ \\
\hline $\mathrm{Pal} 14$ & 0.75 & 69.9 & 50.6 & -4.8 & 4.33 & 1.60 & - & - & - & KM \\
\hline NGC 6093 & 1.95 & 3.1 & 2.7 & -7.8 & 5.54 & 0.80 & - & $49^{\circ}$ & $21^{\circ}$ & $\mathbf{K M}$ \\
\hline NGC 6101 & 0.80 & 11.6 & 4.4 & -6.2 & 4.88 & 1.90 & 0.030 & $44^{\circ}$ & $45^{\circ}$ & KM? \\
\hline NGC 6121 & 1.70 & 6.8 & 0.6 & -6.9 & 5.15 & 1.75 & 0.132 & $10^{\circ}$ & $18^{\circ}$ & $\mathbf{K M}$ \\
\hline NGC 6139 & 1.75 & 2.9 & 1.0 & -7.7 & 5.49 & 1.10 & - & $7^{\circ}$ & $19^{\circ}$ & $\mathrm{KM}$ \\
\hline NGC 6144 & 1.30 & 2.9 & 2.6 & -6.8 & 5.14 & 1.75 & 0.045 & $69^{\circ}$ & $18^{\circ}$ & $\mathrm{KM}$ \\
\hline NGC 6171 & 1.60 & 3.9 & 2.4 & -6.8 & 5.13 & 1.60 & 0.241 & $40^{\circ}$ & $23^{\circ}$ & $\mathrm{KM}$ \\
\hline NGC 6205 & 1.35 & 8.9 & 4.6 & -8.5 & 5.82 & 1.65 & - & $67^{\circ}$ & $67^{\circ}$ & $\mathrm{KM}$ \\
\hline NGC 6218 & 1.70 & 5.0 & 2.3 & -7.5 & 5.39 & 1.40 & - & $72^{\circ}$ & $30^{\circ}$ & $\mathrm{KM}$ \\
\hline NGC 6229 & 1.40 & 30.9 & 20.4 & -8.2 & 5.67 & 1.00 & - & $66^{\circ}$ & $78^{\circ}$ & $\mathrm{KM}$ \\
\hline NGC 6235 & 0.80 & 2.3 & 2.2 & -5.9 & 4.77 & 1.40 & 0.132 & $75^{\circ}$ & $14^{\circ}$ & $\mathrm{KM}$ \\
\hline NGC 6254 & 1.60 & 5.3 & 1.8 & -7.5 & 5.39 & 1.70 & - & $35^{\circ}$ & $27^{\circ}$ & $\mathrm{KM}$ \\
\hline NGC 6256 & - & 2.0 & 0.5 & -4.9 & 4.36 & - & - & - & - & PCC \\
\hline $\mathrm{Pal} 15$ & 0.60 & 62.3 & 28.7 & -5.4 & 4.58 & 1.85 & - & - & - & KM? \\
\hline NGC 6266 & 1.80 & 3.0 & 0.8 & -8.6 & 5.84 & 1.05 & 0.163 & $35^{\circ}$ & $10^{\circ}$ & PCC? \\
\hline NGC 6273 & 1.65 & 2.5 & 1.7 & -9.5 & 6.22 & 1.40 & 0.064 & $59^{\circ}$ & $10^{\circ}$ & $\mathbf{K M}$ \\
\hline NGC 6284 & - & 2.2 & 1.8 & -7.0 & 5.21 & - & - & $86^{\circ}$ & $10^{\circ}$ & PCC \\
\hline NGC 6287 & 1.60 & 2.2 & 1.4 & -6.5 & 5.03 & 1.20 & - & $22^{\circ}$ & $11^{\circ}$ & $\mathrm{KM}$ \\
\hline NGC 6293 & - & 1.6 & 1.0 & -7.1 & 5.23 & - & 0.002 & $25^{\circ}$ & $8^{\circ}$ & PCC \\
\hline NGC 6304 & 1.80 & 2.9 & 0.6 & -7.1 & 5.23 & 1.15 & 0.385 & $1^{\circ}$ & $7^{\circ}$ & KM? \\
\hline NGC 6316 & 1.80 & 4.2 & 1.3 & -8.4 & 5.78 & 1.00 & 0.367 & $83^{\circ}$ & $6^{\circ}$ & $\mathrm{KM}$ \\
\hline NGC 6325 & - & 2.8 & 0.9 & -6.1 & 4.83 & - & 0.124 & $58^{\circ}$ & $8^{\circ}$ & PCC \\
\hline NGC 6333 & 1.60 & 2.2 & 1.4 & -7.7 & 5.48 & 1.20 & - & $90^{\circ}$ & $12^{\circ}$ & KM? \\
\hline NGC 6341 & 1.70 & 9.8 & 4.4 & -8.0 & 5.62 & 0.90 & - & $33^{\circ}$ & $72^{\circ}$ & $\mathrm{KM}$ \\
\hline NGC 6342 & - & 3.4 & 2.0 & -7.2 & 5.27 & 0.0 & 0.329 & $12^{\circ}$ & $11^{\circ}$ & PCC \\
\hline NGC 6352 & 1.10 & 3.4 & 0.8 & -6.5 & 5.02 & 1.70 & 0.319 & $12^{\circ}$ & $20^{\circ}$ & $\mathrm{KM}$ ? \\
\hline $\operatorname{Tr} 2$ & - & 1.4 & 0.4 & -4.9 & 4.37 & - & - & - & - & PCC \\
\hline NGC 6355 & - & 1.8 & 0.7 & -7.3 & 5.33 & - & 0.131 & - & - & PCC \\
\hline NGC 6356 & 1.80 & 8.3 & 3.0 & -8.9 & 5.98 & 1.00 & 0.369 & $2^{\circ}$ & $12^{\circ}$ & $\mathrm{KM}$ \\
\hline NGC 6362 & 1.20 & 5.5 & 2.3 & -6.7 & 5.10 & 1.90 & 0.219 & $52^{\circ}$ & $38^{\circ}$ & $\mathrm{KM}$ \\
\hline NGC 6366 & 1.50 & 5.4 & 1.1 & -4.5 & 4.19 & 2.10 & - & $5^{\circ}$ & $24^{\circ}$ & $\mathrm{KM}$ \\
\hline HP 1 & - & 0.9 & 0.3 & -6.9 & 5.16 & - & - & - & & PCC \\
\hline Liller 1 & 2.05 & 1.2 & 0.0 & -7.9 & 5.58 & - & - & - & - & PCC? \\
\hline NGC 6380 & - & 5.0 & 0.3 & -5.4 & 4.56 & - & - & - & - & PCC? \\
\hline $\operatorname{Tr}=1$ & - & 1.9 & 0.2 & -4.1 & 4.04 & - & - & - & - & $\mathrm{PCO}$ \\
\hline NGC 6388 & 1.85 & 5.6 & 1.6 & -10.0 & 6.40 & 0.80 & 0.300 & $48^{\circ}$ & $16^{\circ}$ & KM \\
\hline Ton 2 & 1.30 & 1.5 & 0.5 & -5.4 & 4.56 & 1.50 & - & - & - & $\mathrm{KM}$ \\
\hline NGC 6397 & - & 6.9 & 0.5 & -5.9 & 4.77 & - & - & $1^{\circ}$ & $25^{\circ}$ & PCC \\
\hline
\end{tabular}


TABLE 1-Continued

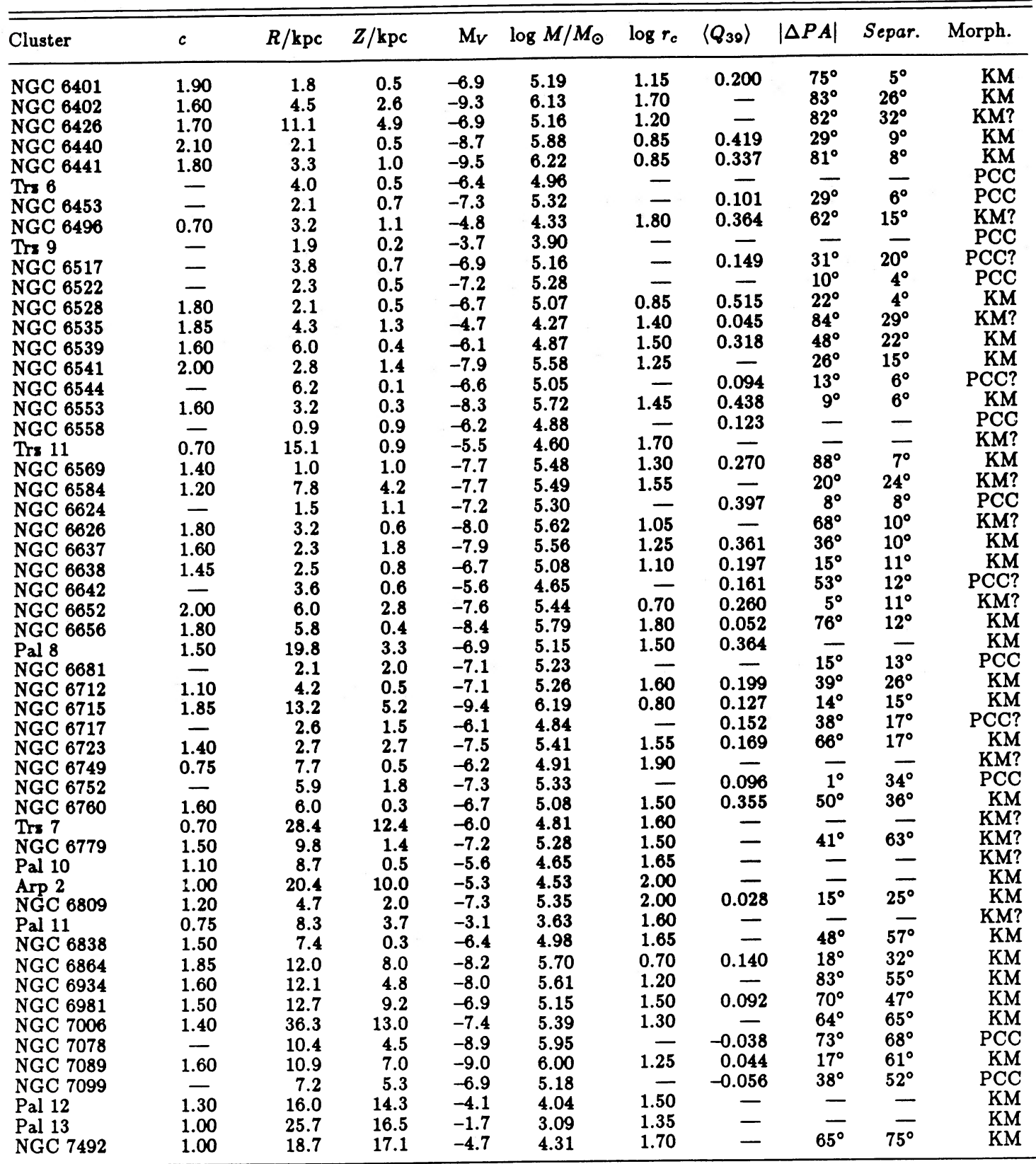

The well-defined minimum near $c=1.6$ and $r_{c}=8$ ".6 leads to the solution displayed in Figure 2 (solid lines) and implies $r_{t}=$ $350^{\prime \prime}$. It is typical of the well-determined fits to King models, that $r_{c}>\theta_{\min }$ and of all fits that $r_{t}>\theta_{\max }$. Very few clusters have any measurements of stellar densities for $\theta>r_{t} / 2$. The formal quality of the model fit is reasonable with $\chi^{2}=24$, for 22 data points. The probability $Q$ that the value of $\chi^{2}$ is that poor is $Q \approx 0.17$, so that one cannot reject it out of hand. For a profile range which is very small, it is usually possible to find a formally good fit, however, the fit may not be well-determined. (See dashed lines in Figs 1 and 2, which illustrate the formal error and best fit when $\theta_{\min }=8.5$.)

Consider the problem of a fit for $\operatorname{Trz} 2$ with $\theta_{\min } \sim 1^{\prime \prime}$, which has been classified as PCC. Figure 3 illutrates the $\chi^{2}$ surface for King model fits over the range $0.25<r_{c}<5^{\prime \prime}$ and $0.25<$ $W_{0}<17.5$. Formally, the best fit within the range has the highest concentration and smallest core radius. However, it is difficult to distinguish any of the high-concentration models with $r_{c}<\theta_{\min }$. This indicates, and a glance at the best-fit cluster profile (Fig. 4) confirms that the core cannot be deduced from the fit to the observations. In this context, PCC clusters are distinguished from other clusters by the fact that their cores are small and, for the time being, that they are unresolved. In practice, an unambiguous, extended, slope $\simeq-1$ power-law section of the surface brightness profile near the center (limited by the seeing disk) was taken as a signature of a postcollapse core (cf. Djorgovski and King 1986).

The $\chi^{2}$ plot shows better agreement as the concentration of the King model increases. If Trz 2 were actually a King model cluster, with a very small unresolved core it must be quite concentrated. A good fit is $r_{c}=0.25$ and $c=3.6$ with $\chi^{2}=7.9$; however, this is not a well-determined fit, as the $\chi^{2}$ surface is 


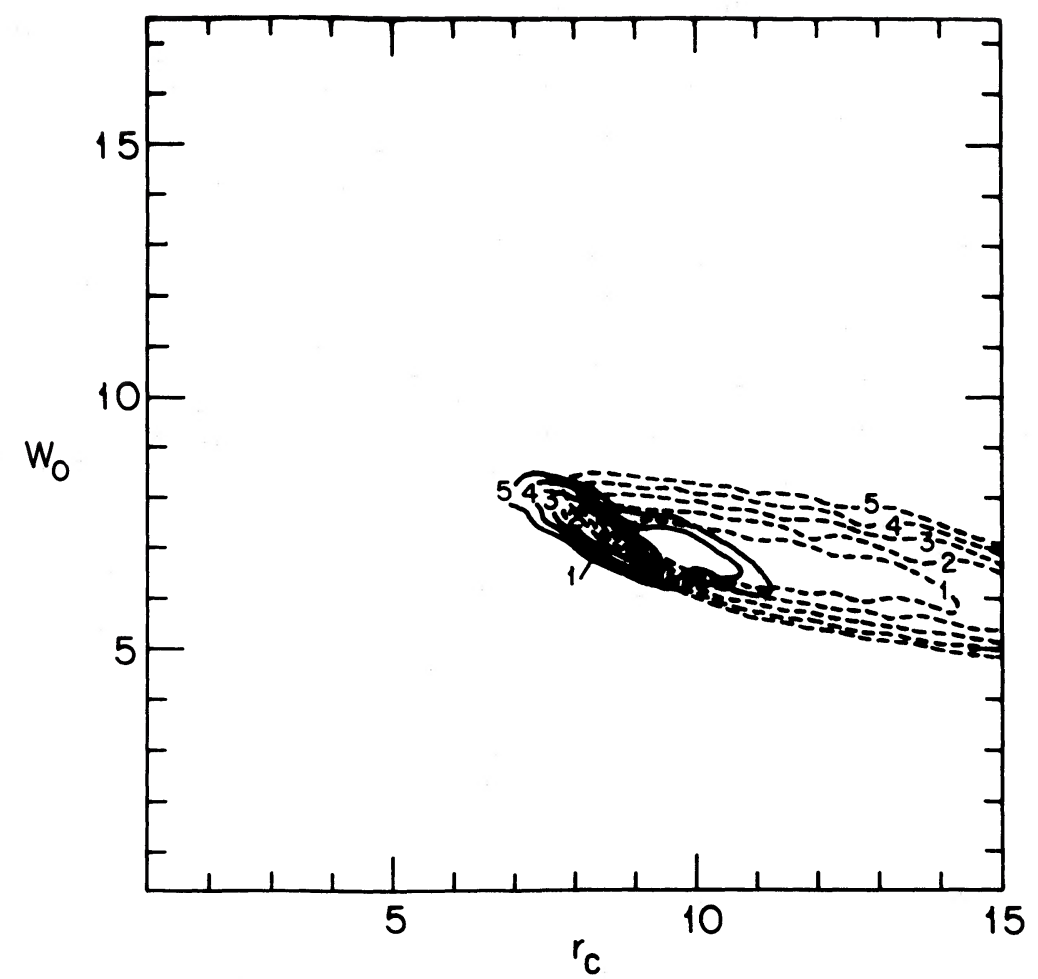

FIG. 1. - $\chi^{2}$ contours for fit to the profile of NGC 6388 as a function of $W_{0}$ and $r_{c}$ for $\theta_{\min }=1.5$ (solid) and $\theta_{\min }=8$ ".5 (dashed). The cluster concentration, $c$, is an increasing function of $W_{0}$. The contours are at $\chi^{2}{ }_{\min }, 2 \chi^{2}{ }_{\min }, \ldots, 5 \chi^{2}{ }_{\min }$ for both the solid and dashed lines, where $\chi^{2}{ }_{\min }=24.8$, the minimum value for the fit with $\theta_{\min }=1.5$. The minimum for the fit with $\theta_{\min }=8.5$ was $\chi^{2}=3.6$, but much flatter.

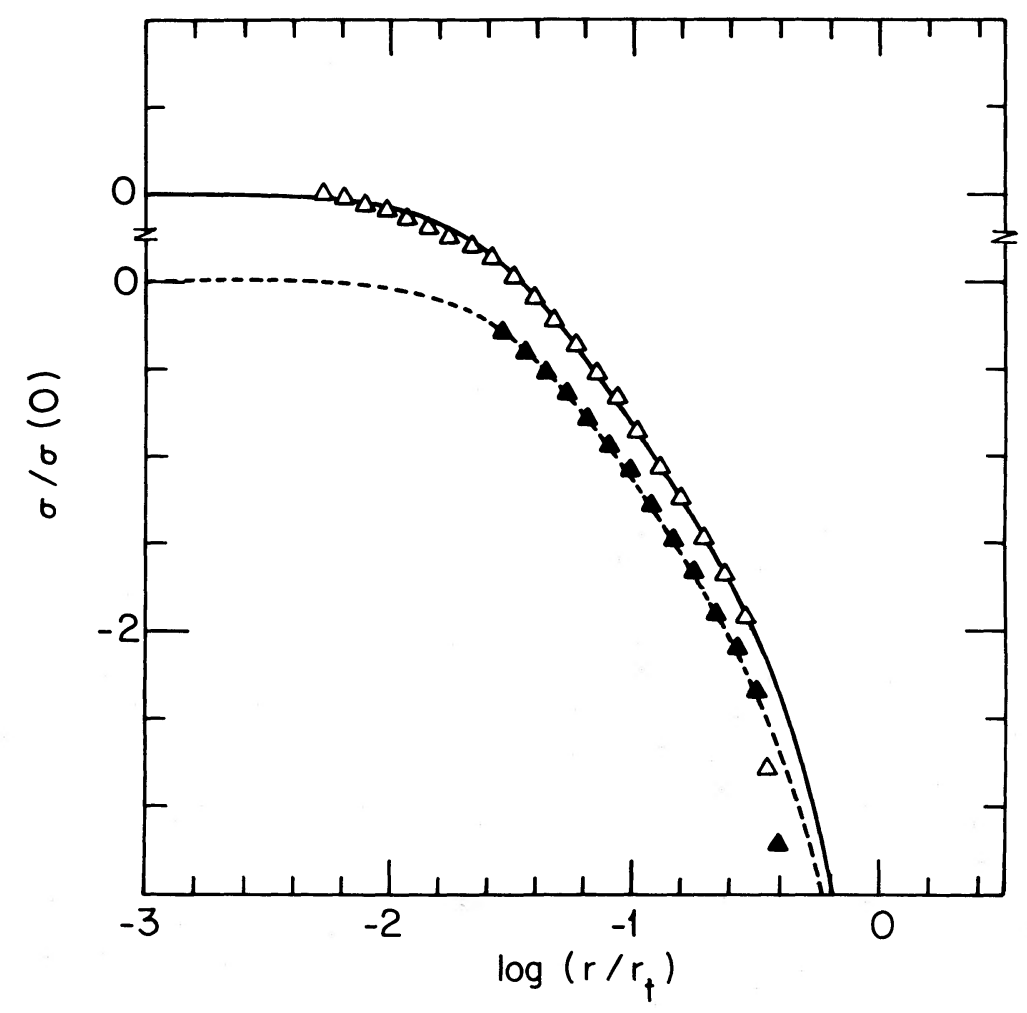

FIG. 2.-NGC 6388 data points and King model fit with $c=1.6$ and $r_{c}=8.6$ for $\theta_{\min }=1 " 5$ (solid line) and $c=1.5$ and $r_{c}=9.6$ for $\theta_{\min }=8$ ".5 (dashed line). 


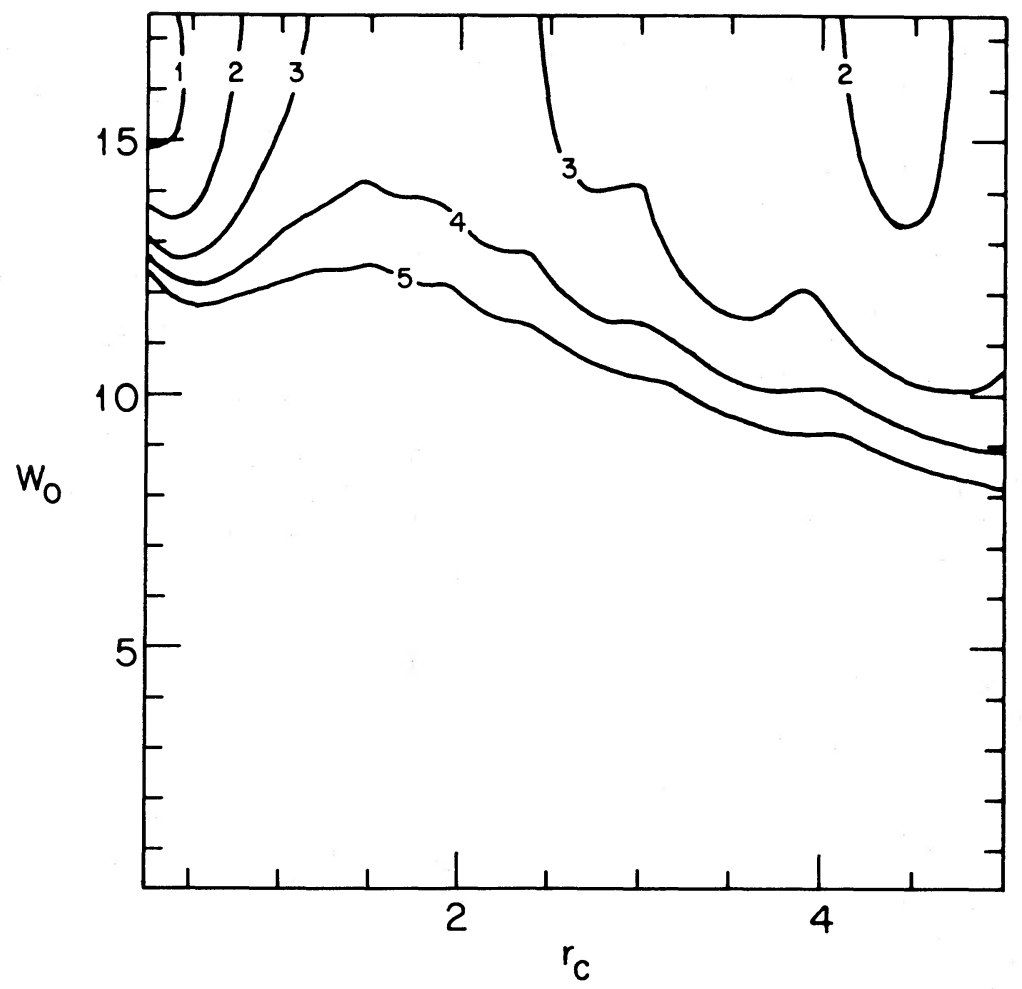

FIG. 3. $-\chi^{2}$ contours for fit to the profile of $\operatorname{Trz} 2$. The minimum displayed point is in the upper left corner with $c=3.6$ and $r_{c}=0$."25 with $\chi^{2}=8.0$, but this is probably not a global minimum.

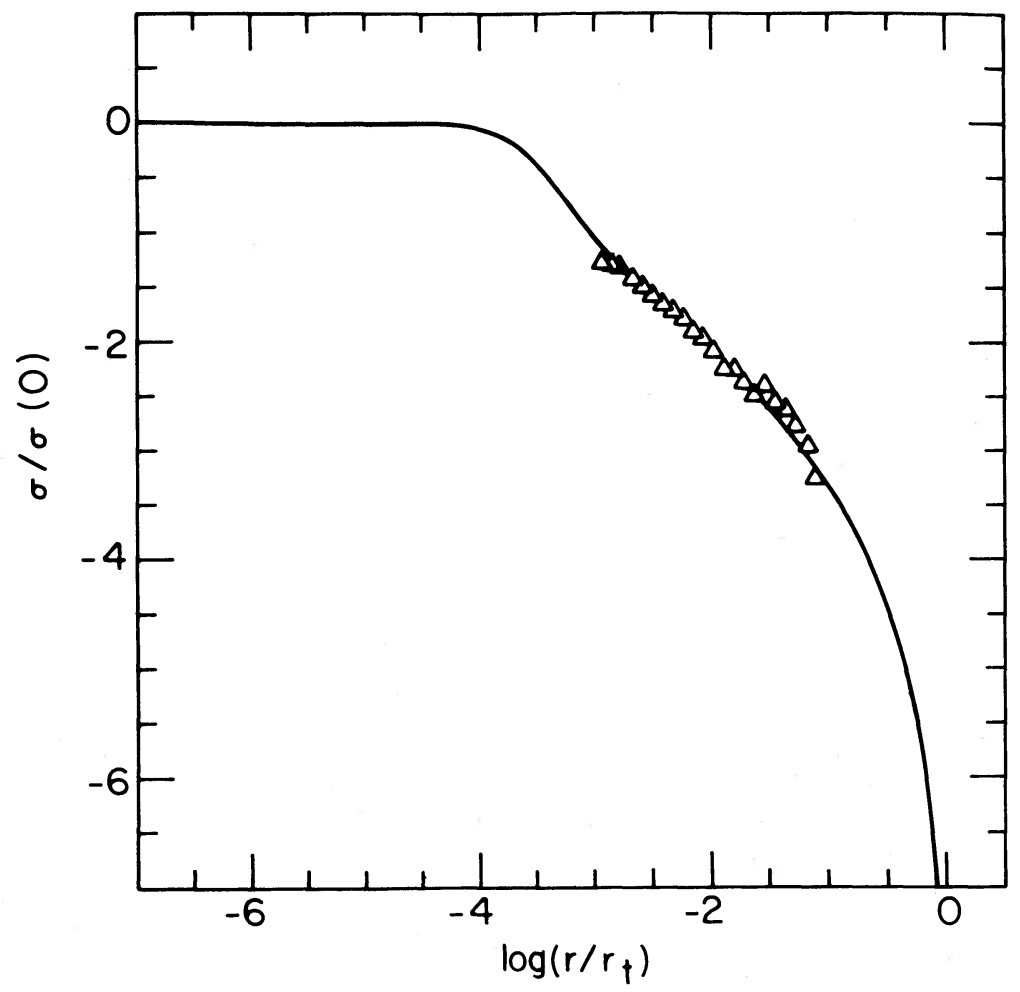

Fig. 4.-Trz 2 data points and best King model fit in the range of Fig. 3, with $c=3.6$ and $r_{c}=0$."25. Note that the core is not resolved, the points sit on the inner halo which is close to an isothermal sphere, and there are no points within about a factor of 10 of the tidal radius. 
very flat for $r_{c}<0$.'25 and $c>3.6$. As the concentration of a King model increases and the size of the core decreases, then the slope of the inner halo tends toward a single characteristic value, that of a isothermal sphere, which is -1.0 . Without core and tidal data, it is impossible to constrain more strongly either $r_{c}$ or $c$. (Note also that this is consistent with the general conclusion regarding fits to clusters which are a priori King models, discussed above.)

The point of this analysis is to make it plausible that $\mathrm{KM}$ and PCC clusters can be distinguished on the basis of surface density profiles. Additional examples and arguments to this effect are given by Lugger et al. (1987). An important future project is to deal with the various observational biases in a more concrete fashion.

\section{a) Observed Cluster Properties}

In practice, one does not perform the above analysis for each cluster, because the complex decisions that must be made in combining the data from a variety of sources are not easy to rigorize. Instead, for the majority of clusters we simply fit extreme King models and the range of derived concentrations is one estimate of the error. We estimate that the average accuracy of our $c$ measurements is about 0.1 to 0.2 , and the measurements of $\log r_{c}$ about 0.1 . We supplement our morphological classifications with the total visual magnitudes, visual extinction, and heliocentric distances of clusters, based on the data compiled by Peterson (1986), wherever available, or Webbink (1985) for a few remaining clusters. From these data we derived the absolute visual luminosities and converted them to the cluster masses by assuming a universal and constant $M / L_{V}=3$ for all clusters. There may be a tendency to underestimate the luminosities (and thus the masses), because the structure of the outer regions of most clusters is poorly known; often the total luminosity is simply based on the outermost available aperture magnitude. An error in the opposite sense is made by the extra light contributed by unremoved foreground stars. The errors introduced by the assumpton of constant $M / L$, the uncertainties in distance and extinction probably swamp the photometric errors. In any case, the derived mass is merely a rough approximation and is probably the worst known number for each cluster. We have not studied in detail whether the different observer selection effects are correlated with the cluster concentration or its position in the Galaxy. We use the galactocentric radii and $Z$-distances from the Galactic plane listed by Webbink (1985), repeated here in Table 1 for convenience. We used the measurements of cluster major axis position angles (PA) by Shawl and White (1987), from which we derive the absolute PA differences between the cluster major axes, and the vectors pointing toward the Galactic center. ${ }^{3}$ We list these PA differences in Table 1 . Finally, we use the metallicity index $\langle Q 39\rangle$ from Zinn and West (1984), repeated here for convenience.

\section{DISTRIBUTION OF PCC CLUSTERS}

\section{a) Distance-independent Analysis}

We begin by analyzing the azimuthal distribution of the clusters about the Galactic center. Following Frenk and White (1982), we introduce the simple coordinate transformation of

\footnotetext{
${ }^{3}$ Shawl and White (1987) computed the PA differences between the cluster axes and vectors pointing towards the Northern Galactic pole. These angles are not directly relevant for our purposes.
}

galactic coordinates $(b, l)$ to $(v, \omega)$, defined as

$$
\cos \omega=\cos b \cos l
$$

and

$$
\tan v=\tan b \csc l .
$$

The new coordinates $(v, \omega)$ have the following interpretation: $\omega$ is the angular displacement from the Galactic center, and $v$ is the latitude where the Galactic plane $(b=0)$ defines $v=0$. A three-dimensional distribution of clusters, arranged in an arbitrary spherically symmetric way about the Galactic center, is uniformly distributed in the variable $v$. This leads to a simple statistical test for symmetry: compare the distribution of the angle $v$ with a uniform random distribution. Figure 5 illustrates the distribution of clusters $(\mathrm{KM} / \mathrm{KM}$ ? are open triangles; PCC/PCC? are filled triangles). Several lines of constant galactic latitude $(b)$ and constant declination are included to provide orientation.

Another possibility that can be tested is that the intrinsic distribution of clusters is ellipsoid or prolate, with a symmetry axis aligned with the perpendicular to the Galactic disk. Let $\epsilon$ be the characteristic flattening, or oblateness $(\epsilon=b / a$, where $b$ and $a$ are principlal axes in Fig. 5). Although the points $(v, \omega)$ generated by $(b, l)$ are not uniformly distributed in $v$, the points $\left(v^{\prime}, \omega^{\prime}\right)$ generated by $\left(b^{\prime}, l^{\prime}\right)=[\arctan (\tan b / \epsilon), l]$ are uniformly distributed in $v^{\prime}$ for an arbitrary $\epsilon$. The "stretching" removes the intrinsic asymmetry of the three-dimensional distribution. Frenk and White (1982) tested the hypothesis that $v^{\prime}$ was distributed uniformly, or, in other words, that the clusters were randomly drawn from an intrinsically flattened distribution. We have performed a similar analysis for the clusters listed in Table 1 for both KM and PCC clusters.

A key point of the analysis is dealing with the observer selection effects. Recently three new clusters were located, "hidden" very near the Galactic plane (Djorgovski 1987a), which reemphasizes the need to deal carefully with the Galactic obscuration. Frenk and White assumed that the cluster catalogs were complete for $|b|>b_{\text {lim }}$, where $b_{\text {lim }}=5.7$ and $10^{\circ}$ (they performed two separate analyses). Then for each annular strip $\left(\omega^{\prime}\right.$ to $\left.\omega^{\prime}+d \omega^{\prime}\right)$ one tests the hypothesis that the clusters are uniformly distributed in $v^{\prime}$ in that range of $v^{\prime}$ with $b>b_{\text {lim }}$. In our analysis $b_{1 \mathrm{im}}$ is a variable ranging from $0^{\circ}$ to 12.5 . The data are folded into a single quadrant $\left(0<v^{\prime}<\pi / 2\right)$, and we use a one-sided $\mathrm{K}-\mathrm{S}$ test against a uniform distribution in transformed angle. The folding makes the test more sensitive to variations in the original distribution which are ellipsoidal in lowest order; it makes it more difficult to reject other sorts of departure from symmetry. Our results are summarized in Figure 6, which shows, as a function of the cutoff, $b_{\text {lim }}$, and the intrinsic ellipticity, $\epsilon, \mathrm{KS}$ contours to reject the hypothesis that the KM (solid) clusters are uniformly distributed in the transformed angular coordinate. Even as $b_{1 \mathrm{im}}$ varies over a wide range, we can rule out a significantly distorted distribution. At the $90 \%$ confidence level, one finds $0.65<\epsilon<1.4$ for any assumed cutoff with $0<b_{\text {lim }}<12.5$ for the KM clusters.

The dashed line in Figure 6 is the 50\% confidence level for PCC clusters. If one chooses as an a priori reasonable value of $b_{\text {lim }}$ the Frenk and White value, 5.7, then one finds $0.7<\epsilon<1.45$. However, this result is not stable to small changes in $b_{\text {lim }}$ (see Fig. 6). If the PCC clusters are distributed approximately spherically symmetrically, then the large fluctuations in the region enclosed by the $50 \%$ level at small $b_{1 \text { im }}$ are due to selection effects. We will argue that PCC clusters are 


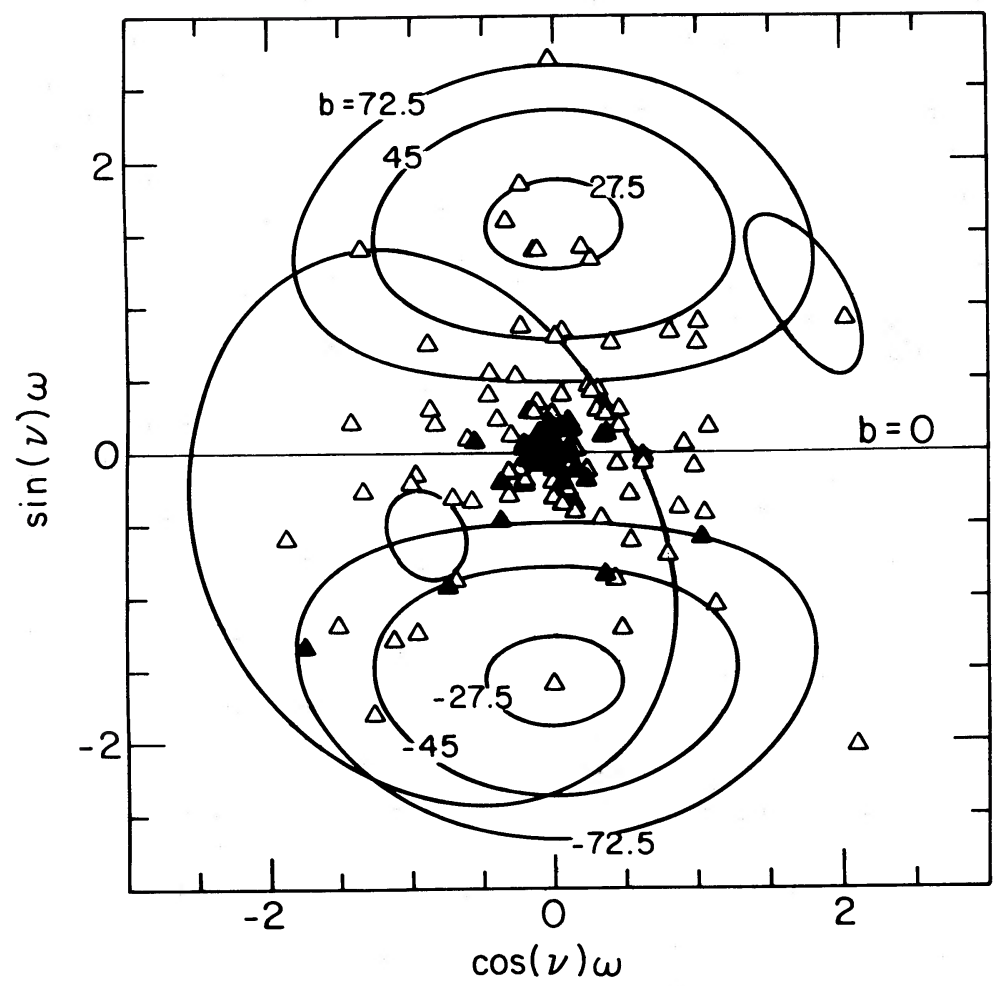

FIG. 5.-The distribution of clusters (KM and KM?: open triangles; PCC and PCC?; solid triangles) about the Galactic center. The two sets of concentric circles are $|b|=27.5,45^{\circ} 0$, and $72^{\circ} .5$. The Galactic plane is the horizontal line. The skewed circles are $\delta=-75.0,0.0$, and 75.0. The assumed ellipsoidal distribution of clusters about the Galactic center has horizontal semiaxis $a$ and vertical semiaxis $b$, with ellipticity, $\epsilon=b / a$.

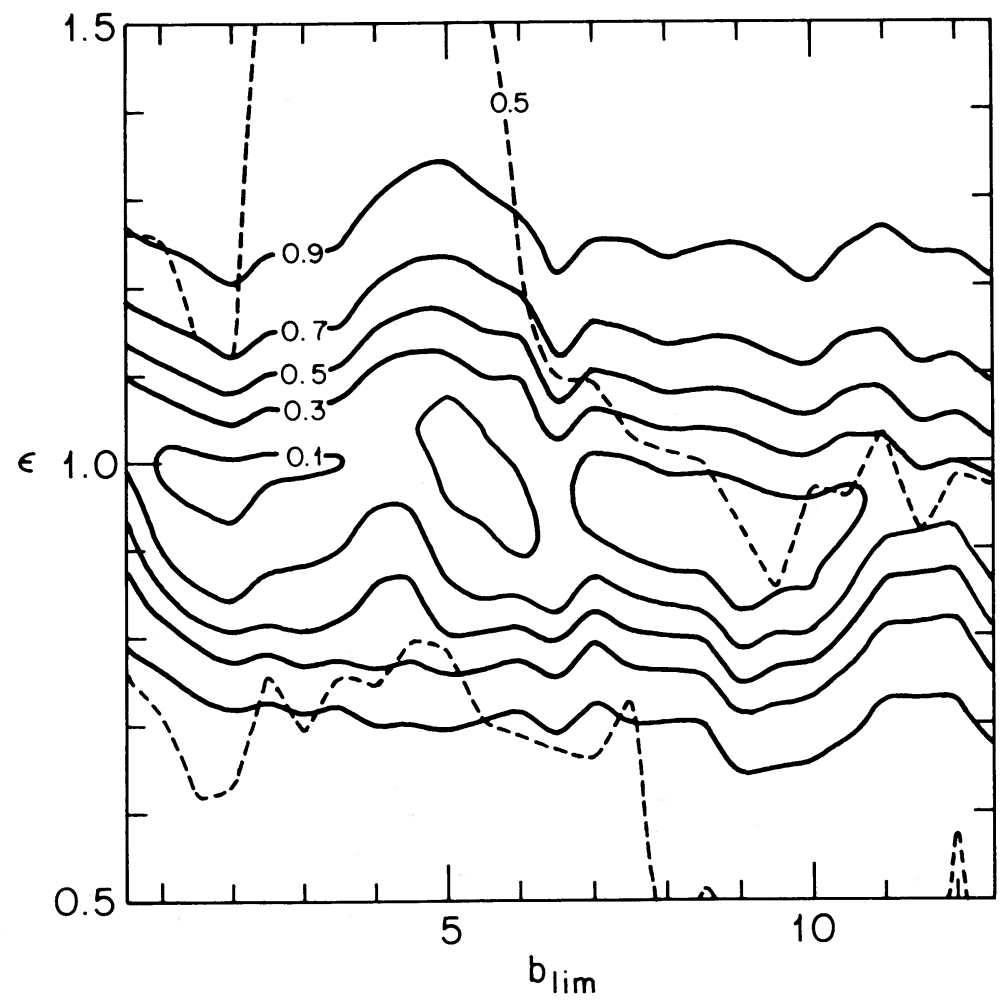

FIG. 6.-KS results to reject the hypothesis that the two-dimensional distribution of clusters have been chosen from a sample with intrinsic ellipticity $\epsilon$ about the Galactic center, with an assumed observational cutoff of $b_{1 \mathrm{lim}}$. The solid lines refer to King model clusters (levels $0.1,0.3,0.5,0.7$, and 0.9 ) and the dashed lines to PCC clusters (level 0.5 ). 
preferentially clustered near the Galactic center. Thus it would not be surprising that they would show a much larger effect due to obscuration from the disk and central bulge than the KM clusters. (We find for $b_{\text {lim }}<7^{\circ}$ that $0.8<\epsilon<2.0$.) Because their number density decreases sharply with distance from the Galactic center, the large deviations in the $50 \%$ confidence level at moderate and large $b_{\text {lim }}$ can be attributed in part to the small number statistics. There are only six clusters left when $b_{\text {lim }}=12.5$. (We find for $12.5>b_{\text {lim }}>7^{\circ}$ that $0.2<\epsilon<1.1$.)

On the other hand, one cannot rule out the hypothesis that the PCC clusters are distributed in an intrinsically nonspherical fashion about the Galactic center. A K-S test of the PCC angular data, without folding into a single quadrant, shows a significantly smaller area consistent with a $50 \%$ confidence level and suggests that the PCC distribution does not have intrinsic ellipsoidal symmetry. In contrast, the KM clusters show a much larger $50 \%$ confidence region and are consistent with the assumption of the symmetry.

We now reduce the data by integrating over $v$, thereby giving a distribution of clusters in $\omega$, i.e., in angular separation from the Galactic center. The cumulative distributions of KM and PCC clusters with $\omega$ is given in Figure 7 for several values of the cutoff, $b_{\text {lim }}=0^{\circ}, 5.7$ and $11: 4$. For each possible cutoff, the cumulative distribution of PCC clusters at the center exceeds the distribution of KM clusters. The number density of PCC and KM clusters have been extracted (smoothing over three and five successive clusters, respectively); the ratio of the densities of PCC and total clusters is given in Figure 8. Our initial assumption was that PCC and KM clusters can be unambiguously distinguished. In addition, all the recently classified PCC clusters are members of existing cluster catalogs and they were included in those catalogs by independent cri- terion. Thus the observational biases in the detection of PCC clusters (subject to above assumption) should be no different than the biases in the detection of other clusters and the ratio of PCC to total clusters should be independent of selection effects. Figure 8 illustrates the ratio for several values of the cutoff, $b_{1 \mathrm{lim}}$ and the same trend is found for each, namely, that there is a greatly increased fraction of PCC clusters at small $\omega$. Hence, PCC clusters are preferentially found near the Galactic center, irrespective of observer selection effects and independent of the uncertainty in distances to individual clusters.

\section{b) Distance-dependent Analysis}

We now assume that cluster distances are reasonably well determined. We expect that the three-dimensional distribution of clusters about the Galactic center will show the trend even more clearly, because there is no line of sight smearing of the cluster density. (The ratio of the number of PCC to total clusters remains independent of selection effects, as discussed above.) Figure 9 illustrates the fraction of PCC clusters at a distance $R$ from the Galactic center, where the threedimensional locations of the clusters are given in Table 1. Figure 10 shows the distributions of galactocentric radii $R$ and distances from the Galactic plane $Z$ for the two morphological categories. As expected, the trend appears with greater signalto-noise ratio than in the two-dimensional projection. However, the distances (and ipso facto the distance-dependent quantities, such as the mass) for the majority of the individual clusters are in general not well known, and we confine the analysis below to looking at the qualitative trends in the binned data. A more sophisticated statistical analysis is not justified by the present quality of the available data.

Table 2 presents the gross properties of the cluster distribu-

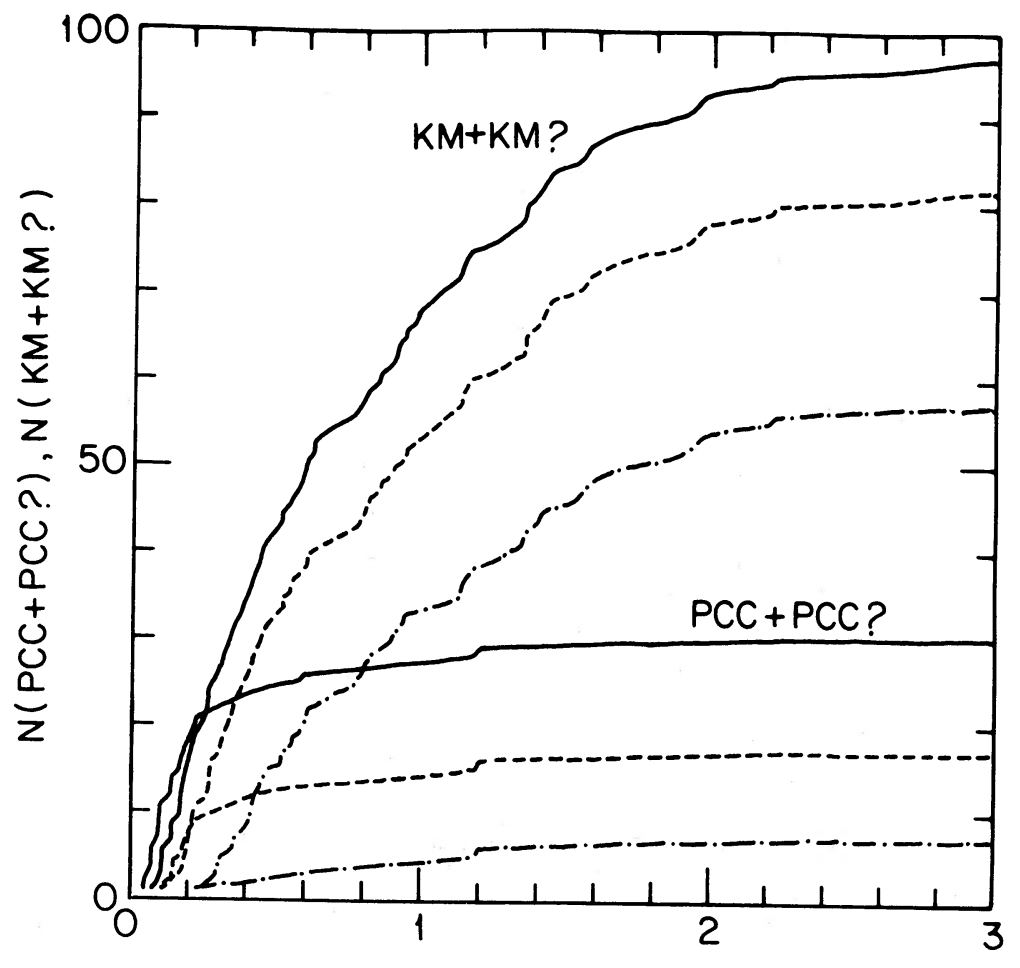

$\boldsymbol{\omega}$

FIG. 7.- Cumulative distribution of King model clusters (top three lines) and PCC clusters (bottom three lines) as a function of the angle of separation $\omega$ from the Galactic center. Three cutoffs in Galactic $b$ have been used, 0.0 (solid), 5.7 (dashed), and 11.4 (dash-dot). 


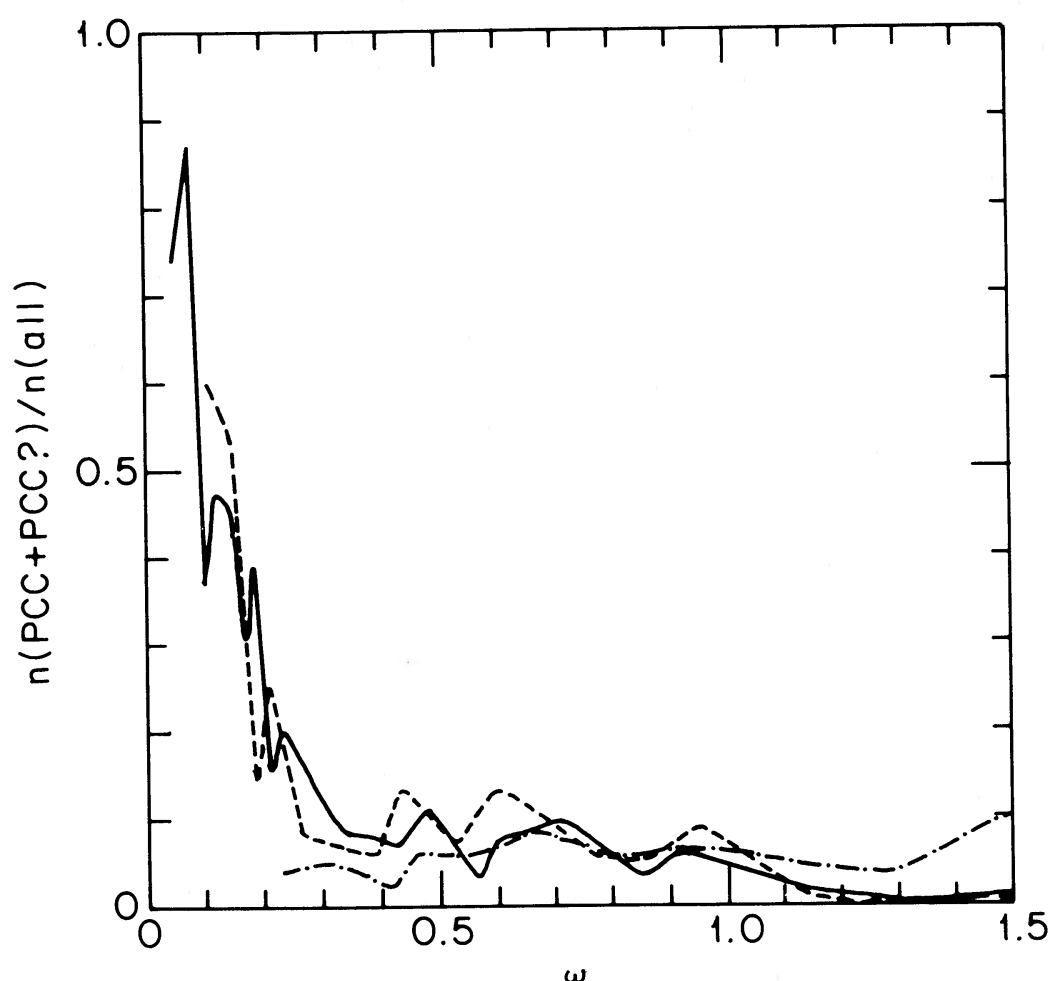

Fig. 8.- Ratio of the surface density of PCC clusters to the surface density of all clusters at an angle of separation from the Galactic center $\omega$ for three cutoffs in Galactic latitude $b$ (as in Fig. 7).

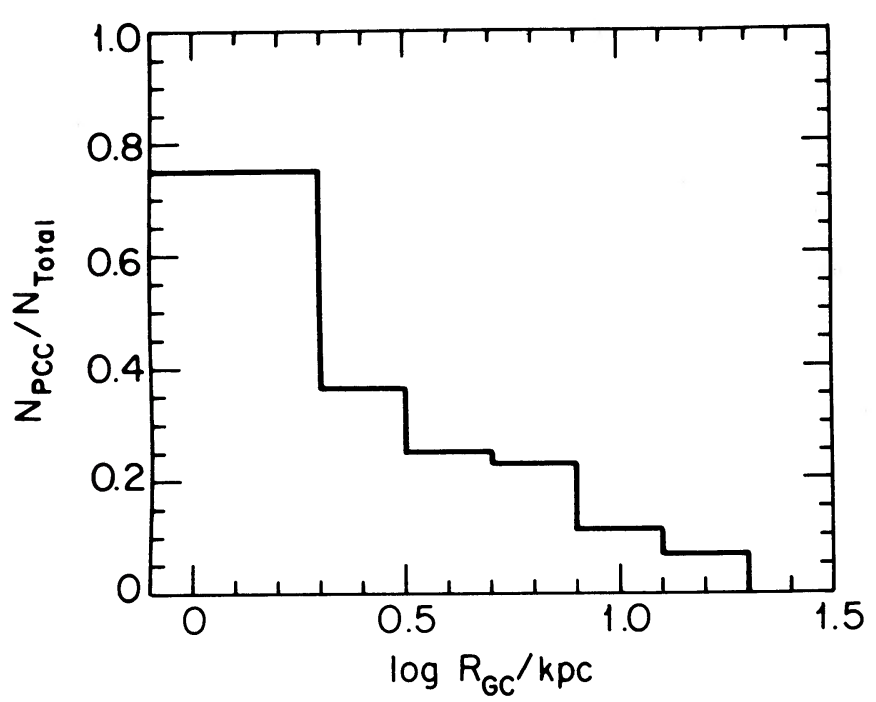

Fig. 9.-Ratio of PCC clusters to all clusters at a distance $R$ from the Galactic center. tion as a function of radial distance from the Galactic center without regard to any selection effects. The complete sample was divided into four subsamples, nearly equal in size, which corresponded to divisions at 3,6 , and $13 \mathrm{kpc}$. The PCC fraction is a very strong function of radius: fully half of the clusters at $R<3 \mathrm{kpc}$ are PCC or PCC?, and the fraction declines monotonically as $R$ increases. Of the clusters that are King models, the trend in average concentration is also noteworthy: the concentration decreases monotonically from 1.59 at $R<3$ $\mathrm{kpc}$ to 1.29 at $R>13 \mathrm{kpc}$. Although $c$ is known to only \pm 0.1 or so for an individual cluster, the expected deviation in the mean for a sample of $N$ clusters is $0.1 / N^{1 / 2} \approx 0.02$, which is much smaller than the size of the trend actually seen. The same trend occurs in the median value of $c$, which is a more robust statistic. On the other hand, the masses do not have a simple behavior with radial displacement from the center. The mass is not well determined for a majority of clusters, so no firm conclusions can be drawn at this time.

As we will discuss in the section on evolution, trends in concentration and in PCC fraction may be related. In addition, from a purely empirical point of view, PCC clusters can often

TABLE 2

Statistics in Galactocentric Radial Bins

\begin{tabular}{|c|c|c|c|c|c|c|}
\hline \multirow{2}{*}{$\begin{array}{c}\text { RADIAL } \\
\text { BIN (kpc) }\end{array}$} & \multirow[b]{2}{*}{$N_{\mathrm{CL}}$} & \multirow{2}{*}{$\begin{array}{c}\text { PCC + PCC? } \\
\text { FRACTION }\end{array}$} & \multicolumn{2}{|c|}{$c(\mathrm{KM})$} & \multicolumn{2}{|c|}{$\log M / M_{\odot}$} \\
\hline & & & Mean & Median & Mean & Median \\
\hline 1. $R<3$ & 32 & 0.50 & 1.59 & 1.60 & 5.13 & 5.22 \\
\hline 2. $3 \leq R \leq 6$.. & 31 & 0.26 & 1.56 & 1.60 & 5.30 & 5.33 \\
\hline 3. $6<R<13$ & 33 & 0.15 & 1.44 & 1.50 & 5.36 & 5.28 \\
\hline 4. $R \geq 13 \ldots \ldots$ & 31 & 0.064 & 1.29 & 1.40 & 4.93 & 4.84 \\
\hline
\end{tabular}




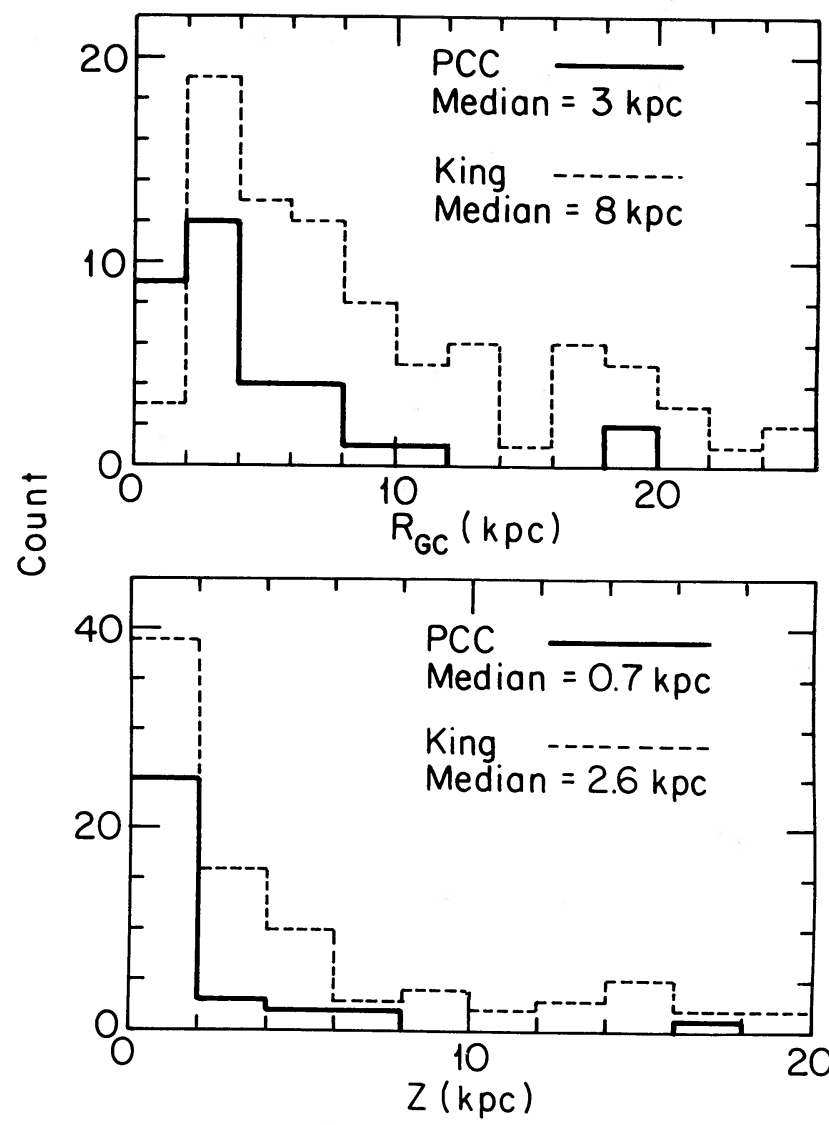

FIG. 10.-Distributions of galactocentric radii, $R$, and distances from the Galactic plane, $Z$, for the clusters with post-core-collapse (PCC) morphology (solid lines), and the clusters with King model (KM) morphology (dotted lines) The PCC clusters are more concentrated toward the Galactic center and plane.

be successfully "fitted" by highly concentrated King models, so it may be appropriate to consider them as simply very high concentration objects. Table 3 divides clusters on the basis of concentration into three classes for the KM clusters, plus one for the PCC clusters. Dependence of some of the quantities of interest on the concentration class is illustrated in Figure 11 (the mean is given by the dotted line, the median by the solid line). Obvious trends in both galactocentric distance and height above the plane exist. The PCCs appear to fit as a simple extension to higher concentration King models in terms of these statistics.

Galactocentric radius $(R)$ and the distance from the Galactic plane $(Z)$ are necessarily correlated, and thus the trends in $Z$ may simply reflect the trends in $R$. However, it is also interesting to look at the behavior of $Z / R$ in different concentration
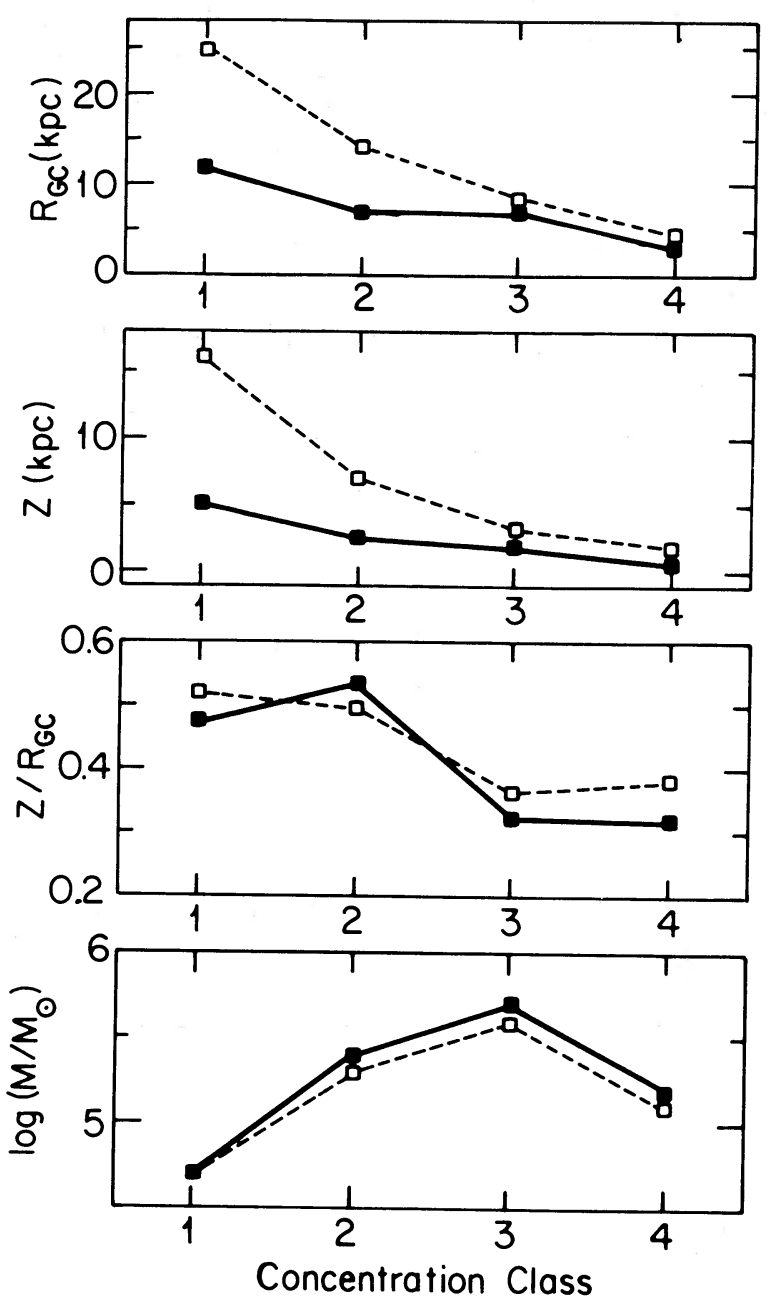

FIG. 11.-Dependences of galactocentric radii, $R$, distances from the Galactic plane, $Z$, the ratios $Z / R_{g}$ and the cluster masses, $M$, on the concentration class, as defined in Table 3 . The mean values for a concentration class are connected by the dotted line, the median values by the solid line.

classes. This quantity is better determined than either $R$ or $Z$ alone, since most of the distance errors cancel. This ratio will on the average be smaller for the less inclined orbits, and vice versa, independent of the shape of the cluster orbit. The more concentrated clusters have, on the average, smaller values of $Z / R$ and thus are preferentially on the less inclined orbits. The trend is uniform from the PCC clusters through the concentration sequence of KM clusters.

There may be a marginal trend within the KM family of mass increase with the cluster concentration; however, that trend does not extend to the PCC clusters taken as the limit of

TABLE 3

Statistics in Concentration Classes

\begin{tabular}{|c|c|c|c|c|c|c|c|}
\hline \multirow{2}{*}{$\begin{array}{c}\text { CONCENTRATION } \\
\text { Class }\end{array}$} & \multirow[b]{2}{*}{$N_{\mathrm{CL}}$} & \multicolumn{2}{|c|}{$R(\mathrm{kpc})$} & \multicolumn{2}{|c|}{$Z(\mathrm{kpc})$} & \multicolumn{2}{|c|}{$\log M / M_{\odot}$} \\
\hline & & Mean & Median & Mean & Median & Mean & Median \\
\hline 1. $c \leq 1.2 \ldots \ldots$. & 28 & 24.8 & 11.8 & 16.1 & 5.2 & 4.7 & 4.7 \\
\hline 2. $1.2<c<1.7$ & 38 & 14.2 & 7.0 & 7.1 & 2.6 & 5.3 & 5.4 \\
\hline 3. $c \geq 1.7 \ldots \ldots \ldots \ldots \ldots \ldots$ & 28 & 8.5 & 6.9 & 3.3 & 1.9 & 5.6 & 5.7 \\
\hline 4. PCC and PCC? ......... & 33 & 4.6 & 3.0 & 1.9 & 0.7 & 5.1 & 5.2 \\
\hline All King models ............. & 94 & 15.7 & 8.0 & 8.7 & 2.6 & 5.2 & 5.3 \\
\hline
\end{tabular}


the concentration sequence. It is tempting to speculate that this effect may reflect an accelerated mass loss from PCC clusters, due to their envelope reexpansion. The cluster masses are very poorly determined, and the effect is clearly not well established at this time.

\section{c) Other Observed Trends}

The slope of the IMF and the range of the stellar mass distribution are important ingredients in determining the time to core collapse. If the recent work of McClure et al. (1986) is confirmed, then metallicity of clusters is tied to the cluster IMF and so to the dynamics of cluster evolution. Here we examine the relationship of the metallicity to the KM and PCC classifications. Zinn and West (1984) give metallicity estimates, expressed as their $\langle Q 39\rangle$ index, for most of the known Galactic globulars. We tested the behavior of the $\langle Q 39\rangle$ for different concentration classes. We find no trend with concentration within the KM family, but there is a marginal difference between the PCC and the KM clusters, in that the PCC ones are on the average metal poorer (Table 4). This small difference persists when we compare the $[\mathrm{Fe} / \mathrm{H}]$ values, which are available for a smaller set of clusters: the mean values of $[\mathrm{Fe} / \mathrm{H}]$ for the PCC and KM clusters are $-1.68 \pm 0.13$ and $-1.44 \pm 0.08$, respectively. This difference is in the opposite sense from what might be expected from the correlation between the cluster concentration and the galactocentric radius (reported above), and the known metallicity gradient among the globulars: more metal-rich clusters are on the average closer to the Galactic center (e.g., the mean $\langle Q 39\rangle$ index changes from 0.127 in our outermost radial bin, to 0.210 for our innermost radial bin). In order to avoid the radial metallicity gradient among the clusters, we computed the statistics of the $\langle Q 39\rangle$ index for the innermost radial bin only $(R<3 \mathrm{kpc})$; the trend with metallicity persists. These results are summarized in Table 4.

Cluster envelopes are the part of the cluster most directly affected by external gravitational perturbations, since they are the most loosely bound part. If clusters were all on circular orbits, then their major axes would align with the vector pointing toward the Galactic center. It is interesting to look at the behavior of cluster envelope orientations with respect to the Galactic center. The absolute position angle (PA) differences between the cluster major axes, and the vectors pointing toward the Galactic center, were derived from the data by Shawl and White (1987) and are listed in Table 1. We formed the $|\triangle \mathrm{PA}|$ histograms for the PCC and KM samples, as shown in Figure 12. There is a weak trend for the PCC clusters to be elongated toward the Galactic center, whereas the KM clusters show no such effect. We emphasize the difficulty of the cluster PA measurements: the clusters are almost round and have bumpy isophotes, and the errors in the evelope PA's must be considerable; these errors and the projection effects would tend to isotropize the observed distributions of $|\triangle \mathrm{PA}|$ and diminish

TABLE 4

Statistics OF The Metallicity INDEX $\langle Q 39\rangle$

\begin{tabular}{crccc}
\hline \hline Cluster Sample & $N_{\text {CL }}$ & Mean & Median & $\sigma($ Mean) \\
\hline All PCC $\ldots \ldots \ldots \ldots \ldots$ & 17 & 0.133 & 0.131 & 0.012 \\
All KM $\ldots \ldots \ldots \ldots \ldots$. & 43 & 0.208 & 0.199 & 0.034 \\
PCC $R<3 \mathrm{kpc} \ldots \ldots .$. & 7 & 0.147 & 0.124 & 0.045 \\
KM, $R<3 \mathrm{kpc} \ldots \ldots$. & 11 & 0.251 & 0.200 & 0.046 \\
\hline
\end{tabular}
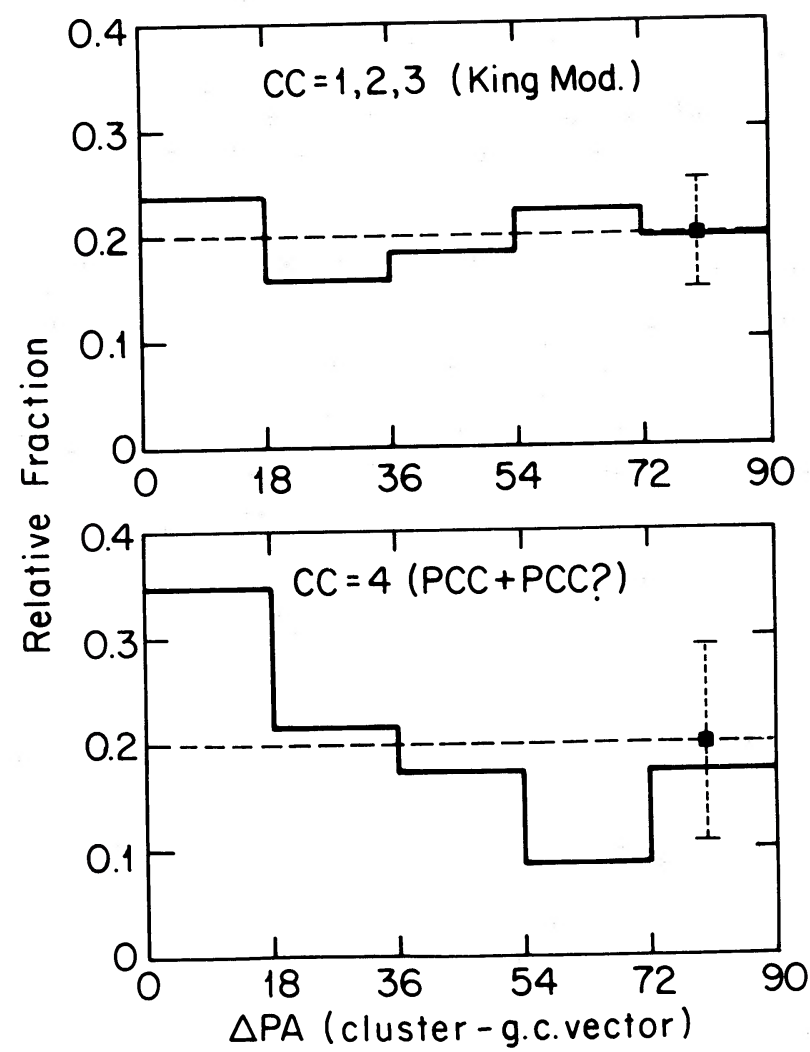

FIG. 12.-Distributions of the cluster envelope orientations, parameterized through the differences between the cluster major axes, and the vectors pointing toward the Galactic center, $|\triangle \mathrm{PA}|$. The PCC clusters (bottom) show a mild trend of pointing toward the Galactic center, whereas the KM clusters (top) show an isotropic distribution. The dashed lines indicate the expected mean count per bin, and the Poisson mean errors are indicated on the right.

the apparent significance of the effect. There is no discernible trend in the envelope ellipticity between the two cluster families.

\section{DYNAMICAL EVOLUTION OF CLUSTERS}

The striking distribution of PCC clusters, and other observed trends, demand an explanation. Recently, Chernoff, Kochanek, and Shapiro (1986) and Chernoff and Shapiro $(1987 a, b)$ considered the large-scale trends in evolution for clusters distributed on circular orbits about the center of the Galaxy. A crude, order-of-magnitude calculation of cluster evolution, which included relaxation and core collapse, tidal shocking, and mass loss by stellar evolution, was carried out. These simulations are described in detail by Chernoff and Shapiro $(1987 a, b)$ and are briefly summarized here. Clusters are assumed to evolve along a sequence of King models. Relaxation drives clusters toward greater central concentration. Since King models have an average density equal to the tidal density, the Galactic location is very important in determining the relaxation time scale for a cluster. Mass loss across the tidal boundary by shocks (also dependent on Galactic location) and/or stellar evolution tends to unbind the cluster and drive it toward lesser central concentrations. The calculation terminates either when the core becomes so concentrated that it begins the self-similar state of gravitational collapse or when the cluster itself becomes so tenuous that the Galaxy's tidal forces destroy it. Recently a complete set of Fokker-Planck 
calculations of multimass cluster evolution with tidal boundary, stellar evolution, and mass loss have been completed (Chernoff and Weinberg 1989). The results support many of the approximate conclusions of these qualitative models including cluster dissolution at low densities and core collapse at high densities.

The principal results of interest from these simulations are shown in Figure 13, which illustrates how initial conditions are mapped into final conditions after about one Hubble time $\left(\approx 1.5 \times 10^{15} \mathrm{yr}\right)$. Consider first the central panel. This summarizes the evolution of a cluster of initial mass $M=10^{5} M_{\odot}$ with a power-law initial mass function (IMF) of slope $\alpha=3.5$ where $d N(M) \propto M^{-\alpha}$, with lower and upper cutoffs of 0.35 and
14.0 $M_{\odot}$, respectively. The abscissa and ordinate of the panel represent the galactocentric radius and the initial concentration (or $W_{0}$ ) for the cluster. Note that the panel is divided into four general areas. Clusters are classified by their fate after the Hubble time of evolution as (1) King models (denoted by K), (2) collapsing models (C), (3) collapsed models (CC), and (4) dissolved models (D). As the clusters evolve along the King sequence, some clusters become dense enough to begin core collapse. Core collapse takes about 330 central relaxation times to complete, so clusters may either be in collapse or have completed collapse (formally, $\rho \rightarrow \infty$ in a finite amount of time). If the clusters become too tenuous they can be destroyed by the tidal field, which occurs very quickly (that is, within a



FIG. 13. - The state of a cluster after a Hubble time is displayed as a function of the initial conditions (galactocentric radius $R$, initial King $W_{0}$ ). Each panel within the plot summarizes the evolution for a given initial mass $\left(10^{4}, 10^{5}\right.$, and $10^{6} M_{\odot}$ across the top) and IMF power-law $\alpha(2.35,3.5$, and 4.5$)$. As discussed in the text, the final states are divided into King models (K), collapsing clusters (C), collapsed clusters (CC), and dissolved clusters (D). 
dynamical time) when the King concentration parameter reaches $W_{0}=0$. Some clusters do not reach either extreme after the given length of time and are still members of the King sequence after a Hubble time. The theoretical question of what process halts the collapse is quite complicated; binary formation is thought to play a crucial role. In any case, we do not attempt to describe the evolution beyond core collapse, and instead we assume implicitly that a cluster that has completed core collapse can be distinguished by its power-law inner core from other clusters. Other panels cover different initial masses $\left(10^{4}\right.$ and $\left.10^{6} M_{\odot}\right)$ and different IMFs $(\alpha=2.35$ and 4.5$)$, as indicated in the figure caption.

All results in Figure 13 are for King models on circular orbits of a given radius about the Galactic center. It is quite likely that some clusters have circular or nearly circular orbits, but how many are on elliptic orbits is unknown and may be a crucial factor in the evolution of the globular system. The actual distribution of initial conditions of the cluster population is unknown, and we are unaware of any reason that they should start out as King models. Nonetheless, the basic trends in cluster evolution in the figure are highly suggestive. Mass loss from clusters by stellar evolution is important for all clusters with small $\alpha$. Clusters close to the Galactic center evolve by relaxation quickly because of the high average density; clusters far from the center evolve by relaxation very little. At intermediate radii $(5 \mathrm{kpc})$ tidal shock can unbind the less dense clusters. Detailed analysis shows that tidal shocks can also increase the rate of core collapse for clusters at intermediate radii; however, the primary determinant of the rate of evolution for most clusters is galactocentric radius.

In principle, it is possible to evolve an arbitrary distribution function of clusters in initial mass, concentration, IMF, and galactocentric radius forward in time and compare with today's distribution. However, we feel that at the moment we are so ignorant of the cluster initial conditions, such a project would appear premature. (One alternative approach has been taken by Aguilar, Ostriker, and Hut [1988], who have searched for a self-consistent description of the present-day cluster distribution function using our new data [Table 1] and known destructive mechanisms.) In addition, the physics of cluster evolution for noncircular orbits has only just begun to be explored. Instead, let us examine some of the trends we have found in the cluster data.

1. The distribution of PCC clusters is peaked toward the Galactic center. The diagrams illustrate that for fixed initial cluster mass, concentration, and IMF power law, that core collapse proceeds most quickly at the center of the Galaxy. In addition, for clusters which do not collapse tidal dissolution occurs more quickly near the center of the Galaxy. Since this is true for every set of initial conditions, it must also be true for any distribution of initial conditions, which are independent of the galactocentric radius, i.e., for a very broad range of initial conditions, PCC clusters will be found preferentially at the Galactic center. A glance at the diagram shows that very strong variations with galactocentric radius of initial mass, concentration, and/or IMF are necessary to change this trend. An immediate corollary is that King model clusters at small galactocentric radius, with small mass, are unexpected. Those that exist may be interlopers, i.e., clusters not on circular orbits, or perhaps they may have collapsed and reexpanded. A better examination of their cores and their three-dimensional velocities are important observational projects for the future.

2 . King models are more concentrated at small galactocen- tric radius than at large radius. The reasons are the same as above, but the trend is less strong. The concentration of a model today is tied to the initial concentration and variation in the initial concentrations is reflected in variation in presentday values. (In contrast, all clusters at small galactocentric radius may have collapsed for a similar range of initial conditions.) In addition, variations in initial mass and IMF strongly effect concentration evolution. Thus, even if all clusters started with exactly the same $c$ but with different masses, then there would be variation today in $c$.

3. Clusters with steep IMFs (few massive stars) undergo core collapse more quickly than clusters with shallow IMFs because of the degree of mass loss. This is true for any initial mass, concentration, and galactocentric radius. (The likelihood of survival during the periods of mass loss is enhanced if the IMF is shallow.) PCC clusters are metal-poor, and, if the McClure et al. (1986) trend is general, then PCCs may have a steep IMF. This would be consistent with their survival and their early collapse.

\section{v. CONCLUSIONS}

One outstanding problem implied by the observations is that there are many clusters which show no traces of the PCC morphology, but have short enough dynamical times to have collapsed in a small fraction of the Hubble time (Djorgovski and King 1986; Djorgovski 1987b). In our sample, between $10 \%$ and $20 \%$ of clusters unambiguously classified as King models have short enough relaxation times $\left(t_{\mathrm{rc}}<3 \times 10^{7} \mathrm{yr}\right.$, and/or $t_{\mathrm{rh}}<3 \times 10^{9} \mathrm{yr}$; these numbers may be uncertain by about a factor of 2 or 3), to have collapsed by now. Some outstanding examples include, e.g., NGC 1851, 5824, 6093, $6341,6440,6528$, or 6864 . The mean and median concentration of these clusters is about $c \approx 1.8$, and they do not differ in their galactocentric radial distribution from the remaining clusters in the same concentration range. The theories of postcollapse evolution of single species cores suggest that the clusters essentially stay in the PCC state "forever," that is, they do not reassume a King model shape in a Hubble time (cf. Goodman 1984 or Cohn 1985). Why there are so many clusters on the verge of collapse has never been adequately answered. One possibility is that the reexpansion theory is wrong, and that the clusters reexpand to a King model state more quickly than the single-species models predict. Another possibility is that the clusters collapse and reexpand in a stochastic manner, due to ejection and formation of central binaries, which stabilize the clusters and provide the energy for reexpansion (McMillan and Lightman 1984). Possibly the postcollapse cores oscillate and are not recognizable at most times (Bettwieser and Sugimoto 1984). All these explanations appear unlikely in the light of our findings: if the clusters recover from the core collapse due to some internal mechanism, the observed dependences of the PCC clusters on the position in the Galaxy are hard to explain. It is possible that these puzzling clusters on the verge of collapse $d o$ have power-law density cusps in their centers, but are composed of dark, heavy stellar remnants, whose distribution is not followed by the low-mass red giants which provide practically all the light (Larson 1984). The explanation of these "uncollapsed" clusters remains a challenge to the theory.

In any case, we have demonstrated that some of the structural properties of globular clusters are strongly dependent on their position within the Galaxy. This relation between a global variable (galactocentric radius) and an internal property (having a small core) follows theoretically from the shorter 


\title{
CHERNOFF AND DJORGOVSKI
}

relaxation times that a tidally limited cluster has at small galactocentric radius, and to a lesser extent, from the interaction of a cluster with the disk. The study of clusters in the Magellanic Clouds, where the average tidal field strength and tidal shocks are weaker than in our own Galaxy, will be useful. In addition, the Magellanic clusters have a larger range in the ages and dynamical time scales than do their Galactic counterparts. Preliminary results by Mateo (1987) and Meylan and Djorgovski (1987) indicate that the frequency of PCC clusters may indeed be smaller in the Clouds.

We are very thankful to the staff of Cerro Tololo Inter- american Observatory for their help in obtaining the data used here. The Berkeley globular clusters survey was conducted in collaboration with Ivan R. King, who generously allowed us to use the data for this work and provided us with much good advice and many stimulating discussions. We also thank Abe Oren, Carl Vuosalo, and Howard Penner for their help in data reductions. We acknowledge helpful conversations with Piet Hut, Gerry Quinlan, Ira Wasserman, and Martin Weinberg.

D. C. acknowledges support by NSF grant no. AST-8415162 and AST-86-57467 at Cornell University. S. D. wishes to acknowledge partial support from Harvard University and California Institute of Technology.

\author{
Aguilar, L., Ostriker, J., and Hut, P. 1988 Ap. J., 335, 720. \\ Bettwieser, E., and Sugimoto, D. 1984, M.N.R.A.S., 208, 493. \\ Chernoff, D., Kochanek, C., and Shapiro, S. 1986, Ap. J., 309, 183. \\ Chernoff, D., and Shapiro, S. 1987a, Ap. J., 322, 113. \\ . 1987b, in IAU Symposium 126, Globular Cluster Systems in Galaxies, \\ ed. J. Grindlay and A. G. D. Philip (Dordrecht: Reidel), p. 283. \\ Chernoff, D. F., and Weinberg, M. D. 1989, Ap. J., submitted. \\ Cohn, H. 1985, in IAU Symposium 113, Dynamics of Star Clusters, ed. \\ J. Goodman and P. Hut (Dordrecht: Reidel), p. 161. \\ Djorgovski, S. 1987a, Ap. J.(Letters), 317, L13. \\ . 1987b, in IAU Symposium 126, Globular Cluster Systems in Galaxies, \\ ed. J. Grindlay and A. G. D. Philip (Dordrecht: Reidel), p. 333. \\ Djorgovski, S., and King, I. R. 1984, Ap. J. (Letters), 277, L49. \\ . 1986, Ap. J. (Letters), 305, L61. \\ Djorgovski, S., King, I. R., Vuosalo, C., Oren, A., and Penner, H. 1986, in IAU \\ Symposium 118, Instrumentation and Research Programmes for Small Tele- \\ scopes, ed. J. Hearnshaw and P. Cottrell (Dordrecht: Reidel), p. 281. \\ Djorgovski, S., and Penner, H. 1985, in IAU Symposium 113, Dynamics of Star \\ Clusters, ed. J. Goodman and P. Hut (Dordrecht: Reidel), p. 73. \\ Elson, R., Hut, P., and Inagaki, S. 1987, Ann. Rev. Astr. Ap., 25, 565. \\ Frenk, C. S., and White, S. D. M. 1982, M.N.R.A.S., 198, 173. \\ Goodman, J. 1984, Ap. J., 280, 298. \\ .1987, Ap. J., 313, 576. \\ Goodman, J., and Hut, P., eds. 1985, IAU Symposium 113, Dynamics of Star \\ Clusters (Dordrecht: Reidel) (GH85).
}

\section{REFERENCES}

Grindlay, J., and Philip, A. G. D., eds. 1987, IAU Symposium 126, Globular Cluster Systems in Galaxies (Dordrecht : Reidel), in press.

Hénon, M. 1961, Ann d'Ap., 24, 369.

King, I. R. 1966, A.J., 71, 64.

1975, in IAU Symposium 65, Dynamics of Stellar Systems, ed. A. Hayli

(Dordrecht: Reidel), p. 99.

King, I. R., Hedemann, E., Hodge, S., and White, R. 1968, A.J., 73, 456

King, I. R., et al. 1989, in preparation.

Kron, G., Hewitt, A., and Wasserman, L. 1984, Pub. A.S.P., 96, 198.

Larson, R. 1984, M.N.R.A.S., 210, 763 .

Lugger, P., Cohn, H., Grindlay, J., Bailyn, C., and Hertz, P. 1987, Ap. J., 320, 482.

Mateo, M. 1987, Ap.J.(Letters), 323, L41.

McClure, R., et al. 1986, Ap. J.(Letters), 307, L49.

McMillan, S., and Lightman, A. 1984, Ap.J., 283, 813.

Meylan, G., and Djorgovski, S. 1987, Ap. J.(Letters), 322, L91.

Newell, E. B., and O'Neil, E. J. 1978, Ap. J. Suppl., 37, 27.

Peterson, C. 1976, A.J., 81, 617.

.1986, Pub. A.S.P., 98, 192

Shawl, S. J., and White, R. E. 1987, Ap. J., 317, 246.

Webbink, R. 1985, in IAU Symposium 113, Dynamics of Star Clusters, ed.

J. Goodman and P. Hut (Dordrecht: Reidel), p. 541

Zinn, R., and West, M. 1984, Ap. J. Suppl., 55, 45.

DAVID F. ChernofF: 602 Space Sciences Building, Cornell University, Ithaca, NY 14853 S. DJorgovski: Astronomy Department, Palomar Observatory, 105-24 Robinson Laboratory, California Institute of Technology,
Pasadena, CA 91125 